\title{
Mechanisms and treatment issues for neuropathic pain
}

Edwin D. Dunteman MD MS

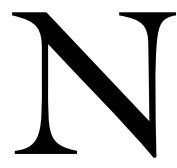

EUROPATHIC pain involves injury or alteration of the normal sensory and modulatory nervous systems to produce a set of symptoms that are often difficult to treat. Multiple changes occur in the injured neural structures themselves, but also in areas not directly injured, often distant from the original insult. The resulting pain complaints include spontaneous "burning" sensations, with intermittent sharp lighteninglike stabbing and lancinating pain. Marked sensitivity and pain may be elicited by minimal stimulation such as a light touch, or even a breeze (allodynia), or temperature changes (thermal hyperalgesia). This paper will serve as a brief introduction and synopsis of material to be presented in a lecture at the annual meeting of the Canadian Anesthesiologists' Society. The primary goal of this effort is to serve as an introduction of what is understood about neuropathic pain processes, as well as the rationale for use of various analgesic drugs, rather than an in-depth review.

Multiple processes are capable of producing sufficient neural injury to produce neuropathic pain (Table). Following the initial injury to a neural component, multiple structural and physiologic processes follow to essentially sensitize the nociceptive pathways, thereby producing amplification and exaggeration of both nociceptive and normally non-painful sensory responses. Normal nociceptive pain serves as a protective function, follows peripheral $\mathrm{C}$ and $\mathrm{A}-\delta$ fibres, and is distinct from the innocuous stimulation, for example, touch which relies on A- $\beta$ pathways. Neuropathic pain, a pathologic process, allows recruitment of $A-\beta$ fibres and the central projections to promote expansion and prolongation of the painful sensations from non-injurious stimuli.

Immediately following a traumatic injury to a peripheral nerve, there is an afferent barrage of activity from that nerve into the central nervous system (CNS), including the dorsal root ganglion, spinal cord dorsal horn, and even brain-stem structures. This ini- tial barrage appears to play a significant role in the 'wind-up' or early sensitization processes, since local anesthesia before the injury inhibits the barrage, and greatly diminishes the longer-term responses that would be expected once the block recedes.

Early pain may begin from persistent spontaneous firing of small afferent fibres at the lesion site. Following Wallerian degeneration of the injured axons, the remaining axon will sprout a growth cone that should follow the intact neurolemma to re-establish the normal function of the nerve. If the axonal sheath is disrupted localized collections of sprouts (neuromas) may develop with enhanced vascularization and sympathetic innervation. These neuromas show sensitivity to substances in the local milieu, such as bradykinin, substance $\mathrm{P}[(\mathrm{sP})$, calcitonin gene related peptide GCRP], glutamate, various prostaglandins, and multiple other substances collectively termed as a "sensitizing soup." Adjacent nerves become involved as more central processes produce a localized antegrade release of sensitizing neurotransmitters (sP, glutamate, CRGP, and 5HT) into uninjured regions to produce 'neurogenic inflammation' and spreading of the 'sensitizing soup.' The neuromas as well as the intact nerve will now develop immature or atypical sodium and calcium channels, which help promote ectopic discharges, spontaneous depolarization, noradrenergic sensitivity, and expansion of the painful receptive field as progression of peripheral sensitization.

Following peripheral nerve injury the dorsal horn experiences multiple changes including death of cells that contain large amounts of gamma-aminobutyric acid (GABA) and glycine, thereby possibly causing a loss of tonic spinal and supraspinal neural inhibition. Additionally, increased numbers of high-threshold $\mathrm{N}$ and $\mathrm{P}$ type calcium channels are expressed in the substantia gelatinosa.

Small afferents fibres (A- $\delta$ and C), carry nociceptive information, and terminate in superficial dorsal horn

From the A \& A Pain Institute of St. Louis, St. Louis, Missouri, USA

Address correspondence to: Dr. Edwin D. Dunteman, A \& A Pain Institute of St. Louis, 456 North New Ballas, Suite 154, St. Louis, Missouri 63141, USA. Phone: 314-692-7246; E-mail: edunte@swbell.net 
TABLE Etiologies of painful neuropathies

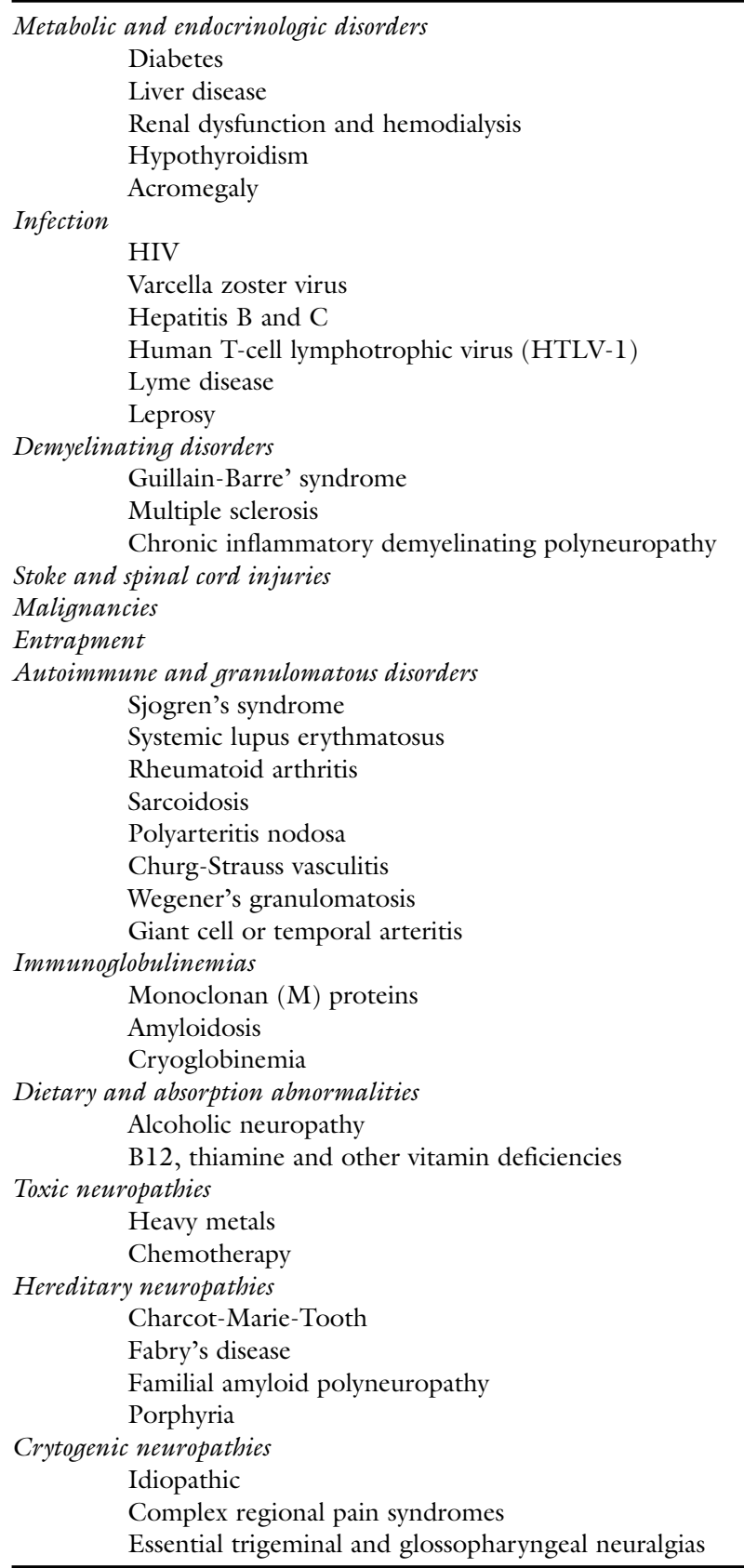

Table modified from Pappagallo M. Neuropathic pain in peripheral neuropathies. In: Tollison CD, Satterthwaite JR, Tollison JW (Eds). Practical Pain Management, 3rd ed. Philidelphia, PA: Lippincott-Williams \& Wilkins; 2002: 431-48.

laminae, no deeper than Rexed II (substantia gelatinosa). Larger afferent Aß fibres terminate in Rexed III and deeper, carrying touch and other innocuous sensations. Following peripheral nerve injury there may be significant sprouting of large afferents up from lamina III into laminae I and II. Thus, low threshold information (touch) is redirected to regions designated for input from high threshold signals (pain). Innocent sensations are now encoded as painful sensations (allodynia).

Glutamate and $\mathrm{sP}$ are the main nociceptive neurotransmitters from $\mathrm{A}-\delta$ and $\mathrm{C}$ afferent fibres. Glutamate activates the $\alpha$-amino-3-hydroxy-5-methlyl-4-isoxazolepropionic acid (AMPA) receptors to prompt an influx of cations $\left(\mathrm{Na}^{+}, \mathrm{K}^{+}, \mathrm{Ca}^{++}\right)$. This depolarization is fast and short-lived. Repeated stimulation may lead to a slower and more sustained depolarizing action potential from the neurokinin receptors. Metabotropic aminocyclopentane-1,3-decarboxylate (ACPD) receptors coupled with inositol phosphate IP3 mobilize microsomal $\mathrm{Ca}^{++}$. Glutamate is also a stimulator of the N-methyl-Daspartate (NMDA) receptor, which also generally requires 'priming' of the receptor via removal of a magnesium ion 'plug' from the associated voltage-gated calcium channel. Once activated, the NMDA receptor/channel complex allows significant calcium influx into the cell cytoplasm from both extracellular and intracellular sources.

Increased intracellular $\mathrm{Ca}^{++}$activates several enzyme cascades including phospholipase $\mathrm{A}_{2}$ (prostaglandin synthesis) and nitric oxide synthase (nitric oxide), each of which promote further responses. Protein kinase C activation phosphorylates NMDA receptors to further increase excitability. Excessive amounts of calcium may also injure the mitochondria and other intracellular organelles. As more of these second-order neurons are gradually depolarized, their responses are increasingly amplified leading to what has become referred to as a 'wind-up' phenomenon, or "central sensitization."

With normal sensory function, Aß fibre activation from low-threshold stimuli cannot activate dorsal horn pain pathways. However, following central sensitization of dorsal horn neurons, innocuous stimulation of Aß fibres may easily activate the pain pathways. Other changes from central sensitization include peripheral receptor field expansion of the secondary neurons, increased magnitude and duration of secondary neuron responses, and reduction of threshold for non-painful stimuli for C-fibre activation to encode and transmit nociceptive information.

Dorsal horn neuronal activity is determined by a balance between excitatory, and inhibitory signals from peripheral, spinal, and descending sites. Peripheral nerve injury may reduce disinhibition via reductions in GABA and activity of various inhibitory interneurons, and down-regulation of opioid receptors in the dorsal horn. With loss of normal inhibitory processes, the CNS receives exaggerated input as 
dorsal horn neurons fire more spontaneously, and with enhanced responsiveness to peripheral inputs.

Many of the pathological processes associated with neuropathic pain may be modulated by drugs currently used for other diseases or syndromes. Despite ongoing research, as well as years of proven clinical efficacy, regulatory "approval" or acknowledged "indications" is delayed, causing many drugs to be used as "offlabel" treatment. Understanding the pathology of neuropathic pain, and the mechanisms of action of various neuromodulating drugs allows the physician to formulate new treatment regimens. Certain antidepressants, antiepileptic, cardiac, and other assorted drugs have been shown beneficial for helping patients with neuropathic pain.

Various antidepressants have been used as adjunctive analgesics for a number of neuropathic pain syndromes by enhancing the central descending pathways. Inhibitory fibres from the Locus Ceruleus use norepinephrine (NE) as a neurotransmitter, while those from the periaqueductal gray use serotonin. Tricyclic antidepressants have been shown useful in reducing pain via noradrenergic and serotonergic pathway. Inhibition of the reuptake of the neurotransmitter from the synapse promotes longer activation, and therefore enhanced descending inhibition of pain signals entering the spinothalamic tract from peripheral nerves. NE reuptake inhibition is thought to be more beneficial than serotonin reuptake inhibition for analgesia. Unfortunately, anticholinergic side effects such as dry mouth, urinary retention, and arrhythmias, among others limit tolerability and analgesia effect. Selective serotonin reuptake inhibitors have shown less benefit as a class, though paroxetine and citalopram have been shown helpful in recent studies. Selective norepinephrine reuptake inhibitors (SNRI; venlafaxin) have shown promise for diabetic neuropathy and other painful syndromes.

Baclofen acts at GABA receptors to enhance the inhibitory chloride channels. Another muscle relaxant, tizanidine enhances presynaptic alpha 2 receptors to inhibit activating neurotransmitter release.

Capsaicin, derived from hot chili peppers activates the peripheral $\mathrm{C}$ fibres on the skin or mucus membranes. Prolonged use leads to decrease in allodynia in the region. At one time it was felt that capsaicin worked by depleting sP. More recently, significant neurolysis of the $s c$ C-fibres appears to be a major mechanism.

Opioids have long been the main analgesic for a wide variety of pain syndromes. Opioid use in neuropathic pain syndromes has been controversial. The same NMDA mediated mechanisms arising from $\mathrm{Mu}$ recep- tor activation that promote opioid tolerance, also promote neuropathic pain. Descending activation signals from opioids appear related to cholecystikin and dynorphins in the brain stem and spinal cord to promote release of glutamate and CGRP in the dorsal horn.

Antiepileptic drugs are gaining considerable use for the treatment of neuropathy pain due to mechanisms that inhibit one or several sites along the pain sensitization pathways. Drugs such as carbamazepine, phenytoin, oxcarbazipine, lamotrigine, topiramate, mexilitine and lidocaine block peripheral sodium channels. Central calcium channels are inhibited by several anti-epileptic drugs (AEDs). Voltage-sensitive Ca channels may be inhibited by felbamate, lamotrigine, and topiramate. T-type Ca channels are blocked by ethosuximide, zonisamide, and possibly valproate. Gabapentin blocks the $\alpha_{2} \delta$ subunit of voltage-sensitive Ca channels.

In addition to channel blockade, topiramate and zonisamide inhibit carbonic anhydrase to possibly produce a localized metabolic acidosis. Such an acidosis may decrease NMDA-mediated excitation, and increase GABA-mediated inhibition.

Other, more novel mechanisms of action by the various newer classes of AEDs appear to be analgesic as well. Levetiracetam block $\mathrm{N}$ type $\mathrm{Ca}^{++}$channels, as well as inhibits the action of beta carbolines and zinc, both of which typically oppose GABA receptor mediated pain inhibition. Beta carbolines also appear active in NMDA activation, but have not yet been characterized. Zonesamide appears to inhibit nitric oxide synthase, as well as scavenge free-radicals that may promote cell membrane injury.

Ketamine, memantine and dextromethorphan have been suggested to be analgesic through their NMDA antagonism. Ketamine, a potent NMDA antagonist often produces unwanted dysphoric side effects. Slow titration, possible co-administered with a sedative may limit the mental status changes. Dextromethorphan is a weak NMDA antagonist that has been shown analgesic primarily at fairly significant doses $\left(>3 \mathrm{mg} \cdot \mathrm{kg}^{-1}\right)$. In order to avoid dry mouth and sedative side effects, slow titration is advised. Commercial cough suppressants are an easy way to initiate therapy, but compounding by a specialized pharmacy is needed as the doses are escalated. Memantine is a new NMDA antagonist recently approved for treatment of dementia and may hold promise for neuropathic pain, though earlier studies are mixed. Interestingly, the opioid methadone also has weak NMDA antagonism traits.

'Rational polypharmacy' has gained widespread acceptance, using more than one type of analgesic 
simultaneously may modulate multiple pain mechanisms to promote additive or synergistic responses. Such a practice is well accepted in such issues as hypertension, oncology and infectious disease. For example, realizing that mechanisms of action differ between an SNRI, a sodium channel antagonist AED, or a calcium channel antagonist AED, many are now adding a complementing agent when single agents produce only a partial response. Ongoing preclinical and clinical investigation is of paramount importance to promote the evolution of our understanding of neuropathic pain mechanisms, and to develop the most effective treatment paradigms.

\section{Selected references}

Adamec RE. Evidence of lasting potentiation of amygdala efferents in the right hemisphere underlies pharmacological stressor (FG-7142) induced lasting increases in anxiety-like behavior: role of GABA tone in initiation of brain and behavioral changes. J Psychopharm 2000; 14: 323-39.

Berde CB. New and old anticonvulsants for management of pain. Technical Corner from IASP Newsletter 1997.

Beydoun A, Backonja MM. Mechanistic stratification of antineuralgic analgesics. J Pain Symptom Manage 2003 ; 25: S18-30.

International Association for the Study of Pain: IASP pain terminology. Classification of pain. In: Mersky H, Bogduk N (Eds). Task Force on Taxonomy, 2nd ed. Seattle: IASP; 1994: 209-14.

Jasmin L, Rabkin SD, Granato A, Boudah A, Ohara PT. Analgesia and hyperalgesia from GABA-mediated modulation of the cerebral cortex. Nature 2003; 424 : 316-20.

Klees TM, Brodtkorb E, Nakken KO, Lossius R, Johannessen $S I$. Clinical experience with the new antiepileptic drug levetiracetam. Acta Neurol Scand 2003; 107: 428.

Olat H, Cesaro P. Pharmacology of neuropathic pain. Clin Neuropharmacol 1995; 18: 391-404.

Pappagallo $M$. Neuropathic pain in peripheral neuropathies. In: Tollison CD, Satterthwaite JR, Tollison JW (Eds). Practical Pain Management, 3rd ed. Philidelphia, PA: Lippincott-Williams \& Wilkins; 2002: 431-48.

Price D. Psychological Mechanisms of Pain and Analgesia. Progress in Pain Research and Management, vol 15. Seattle, WA: IASP; 1999.

Rigo JM, Hans G, Nguyen L, et al. The antiepileptic drug levetiracetam reverses the inhibition by negative allosteric modulators of neuronal GABA- and glycinegated currents. Br J Pharmacol 2002; 136: 656-72.

Siddall PJ, Cousins MJ. Introduction to pain mechanisms: implications for neural blockade. In: Cousins MJ,
Bridenbaugh PO (Eds). Neural Blockade In Clinical Anesthesia and Management of Pain, 3rd ed. Philidelphia, PA: Lippincott-Raven; 1998: 675-713. Sindrup SH, Jensen TS. Efficacy of pharmacological treatment of neuropathic pain: an update and effect related to mechanism of drug action. Pain 1999; 83: 85-6.

White HS. Comparative anticonvulsant and mechanistic profiles of the established and newer antiepileptic drugs. Epilepsia 1999; 40(suppl 5): S2-10.

Woolf CJ, Mannion RJ. Neuropathic pain: aetiology, symptoms, mechanisms, and management. Lancet 1999; 353: 1959-64.

Ossipov MH, Lai J, Vanderah TW, Porreca F. Induction of pain facilitation bt sustained opioid exposure: relationship to opioid antinociceptive tolerance. Life Sci 2003; 73: 783-800. 